\title{
Article \\ Research on Microstructure Characteristics of Welded Joint by Magneto-Optical Imaging Method
}

\author{
Congyi Wang (D), Chuncao Wang, Xiangdong Gao*, Meng Tian and Yanxi Zhang
}

Guangdong Provincial Welding Engineering Technology Research Center, School of Electromechanical Engineering, Guangdong University of Technology, Guangzhou 510006, China; wangcongyi126@126.com (C.W.); 18680529185@163.com (C.W.); t15203801802@163.com (M.T.); yanxizhang@gdut.edu.cn (Y.Z.)

* Correspondence: gaoxd@gdut.edu.cn; Tel.: +86-137-1145-7326

\begin{abstract}
This paper proposes an approach for analyzing the microstructure evolution of laser welding seam by magneto-optical imaging (MOI). The Faraday magneto-optical effect and magnetic domain theory are used to account for the MOI mechanism. The influence of laser welding on the welded joint was inspected by the analysis of color, grayscale and brightness of magneto-optical images. The relation between the brightness of magneto-optical image and grain size of microstructure is discussed as well, and the characteristics of magneto-optical images of weld microstructure were compared with those of scanning electron microscope (SEM) images. Experimental results show that three regions, including the weld zone (WZ), the heat-affected zone (HAZ), and the base metal (BM) are distributed in the magneto-optical image of the welded joint. The MOI method can investigate the microstructure evolution of welded joints and provide a theory and experimental basis for detecting weld defects.
\end{abstract}

Keywords: laser welding; magneto-optical imaging; microstructure; welded joint

Citation: Wang, C.; Wang, C.; Gao, X.; Tian, M.; Zhang, Y. Research on Microstructure Characteristics of Welded Joint by Magneto-Optical Imaging Method. Metals 2022, 12, 258. https://doi.org/10.3390/met12020258

Academic Editor: Antonio Riveiro

Received: 28 December 2021

Accepted: 26 January 2022

Published: 29 January 2022

Publisher's Note: MDPI stays neutral with regard to jurisdictional claims in published maps and institutional affiliations.

Copyright: (c) 2022 by the authors. Licensee MDPI, Basel, Switzerland. This article is an open access article distributed under the terms and conditions of the Creative Commons Attribution (CC BY) license (https:/ / creativecommons.org/licenses/by/ $4.0 /)$.

\section{Introduction}

Laser welding is widely used in manufacturing with a complicated process [1-6]. During the welding, the welded joint microstructures in different areas inevitably make a big differences under diverse welding conditions [7-9]. The differences of welded joint microstructures directly decide their properties, such as hardness, toughness, and plasticity $[10,11]$. The effect of post weld heat treatment on microstructure and fracture toughness of friction welded joint was studied [12]. Hence, it is necessary to investigate the characteristics of welded joint microstructure [13].

In recent years, the microstructures of welds have been widely observed by electron backscatter diffraction (EBSD), transmission electron microscopy (TEM), X-ray Diffraction (XRD) imaging, metallurgical optical microscopy and SEM [14-17]. EBSD can be used to estimate grain boundary, phase identification, the orientation of grains, texture, and strain [18]. XRD was the first method to study the microstructure of matter and can be used to determine phase, crystallinity, and the lattice constant. TEM can observe the internal microstructure of a weldment [19]. Moreover, the metallurgical optical microscope is used to observe the metallographic structure of opaque objects such as metals and minerals. A light beam from the objective to the surface of the observed object is reflected from the object's surface before returning to the objective image in the metallographic microscope [20]. And the SEM is used for observing the fine structure of the weld with high magnification [21,22]. However, there are shortcomings in these approaches. EBSD needs specialized instruments to make samples. Sometimes, TEM requires the preparation with ultramicrotome in a vacuum chamber. And XRD may be harmful to human because of radiation. Moreover, in most cases, the samples need to be chemically etched by metallographic optical microscopy and scanning electron microscopy, which are inconvenient to use in industrial settings due to their ultra-precision [23,24]. Furthermore, these two 
methods have low detection efficiency, which can't achieve automated detection in industry. Compared with SEM and metallurgical optical microscope detection, MOI technique can be achieved without prior grinding and corrosion in advance and can realize online testing with high detection efficiency. Dozens of images are captured in a second by the MO imaging sensor. Hence, the MO imaging method can provide a large number of samples for analyzing the microstructure of welding seam in intelligent inspection and save a lot of time. Li et al. compared the difference of welded joint microstructures by $\mathrm{MO}$ imaging method, which provided the theoretical basis for researching of weld microstructure by MOI, and the weldment's microstructure was investigated by the optical microscope [25]. However, the magnification of the optical microscope is limited, which can't further analyze and evaluate the microcrystalline evolution caused by welding heat. Gao and Ma et al. investigated the characteristics of $\mathrm{MO}$ images of weld defects under alternating magnetic field excitation [26]. Gao and Du et al. proved that rotating magnetic field excitation had more advantages than alternating magnetic field excitation in detecting multi-directions and subsurface defects by MO imaging method [27].

The MO imaging detection approach is used to research the welded joint microstructure under constant magnetic fields. Magnetic domain theory and the magnetizing properties of ferromagnetic materials were studied to further investigate the application of this approach. The ferromagnetic coil is supplied with a direct-current signal generating a constant magnetic field. This experiment investigates the relationship between the grain size of the material and magnetic induction intensity and analyzes this grain difference in the MO images of the weld microstructure. This approach facilitates human work of sample preparation and acquires information of objective with high efficiency, thus, the cost of investigation is reduced and the production efficiency of industrial automation is improved. The recognition of welding defects requires advanced image processing technology and methods [28]. This method is combined with image edge extraction technology and used to detect weld width in the future [29]. The purpose of this study is to accurately identify the three regions of welded joints through the differences in microstructure magneto-optical imaging.

\section{Magnetic Optical Imaging and Materials}

\subsection{Magneto-Optical Imaging}

Magneto-optical imaging is based on the Faraday magneto-optical effect [30]. When linear light passes through an MO film or magnetic medium, the polarization direction of the linearly polarized light is rotated to a certain angle $\theta$ under the influence of an external magnetic field. The empirical rotation angle of a polarization plane is called Faraday rotation angle (in radians), which can be expressed as

$$
\theta=B V L
$$

where $V$ (in radians per tesla per meter) is the material-specific Verdet constant of the medium, $B$ represents the magnetic induction intensity (in teslas) of the magnetic field paralleling to the direction of polarized light, and $L$ represents the distance (in meters) that the light travels through the magnetic medium. Figure 1 shows the working principle of observing the welded joint microstructure using MO imaging system. As can be observed, the light emitting diode (LED) gives out incident light and the direction of light is the same as the magnetic field direction. Then the incident light turns to be linearly polarized light through the polarizer. The weldment is magnetized by the constant magnetic field generated by the electromagnet, and the magnetic states of the weldment and MO film are changed by the induced magnetic fields. The polarized light passes through the magnetooptical film and reflected by the mirror coating, which caused a Faraday rotation in the polarized light. Afterward, the polarized light, containing the information of the welded joint microstructure, analyzed by the analyzer in the direction of emergent light. Finally, the polarized light is captured by a CMOS camera. 


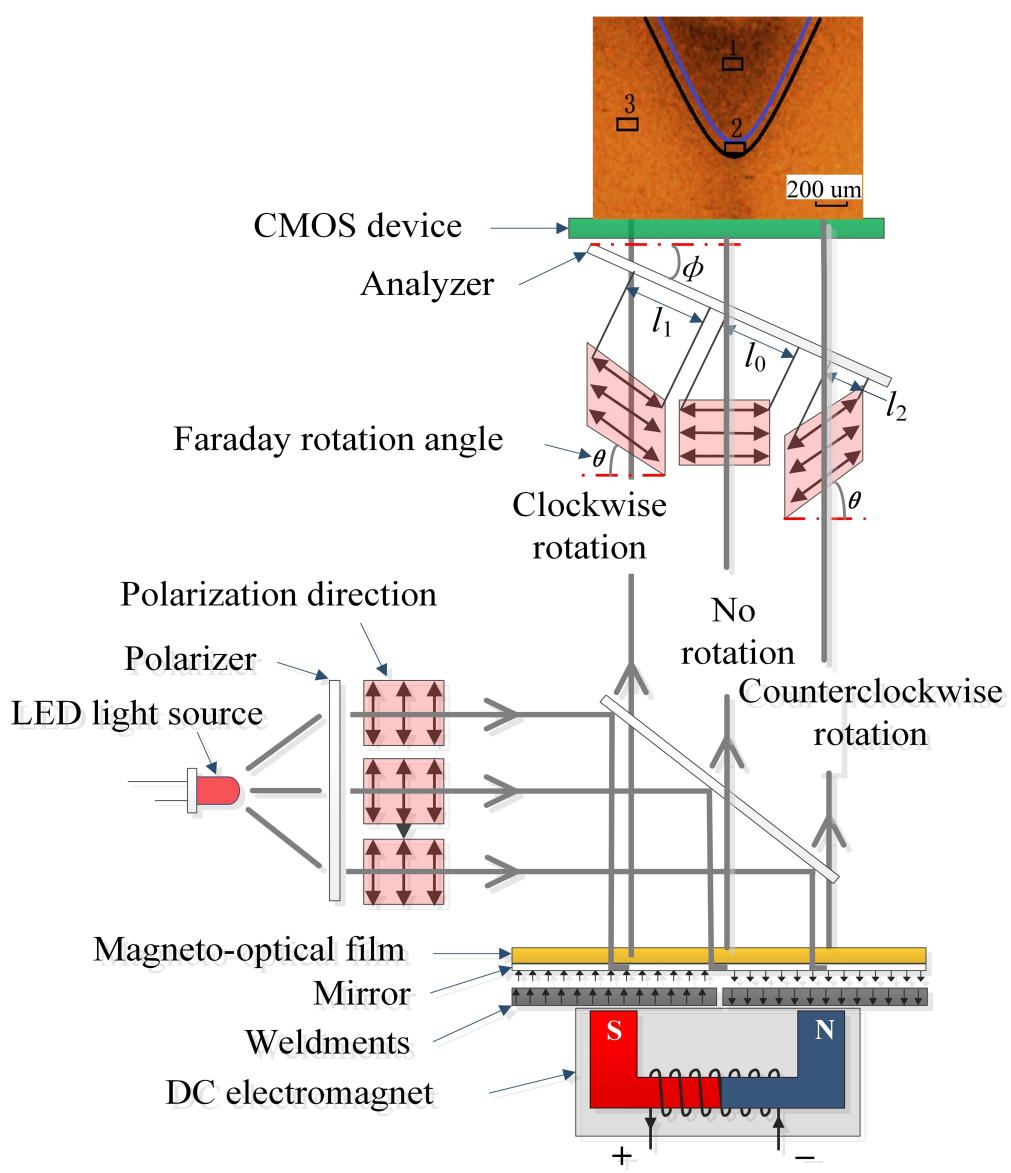

Figure 1. Schematic diagram of magneto-optical imaging.

When the polarized light goes through the MO medium under the constant magnetic field, the reflected light intensity $I_{i}(i=1,2,3)$ can be described as

$$
I_{i}=E^{2} \cos ^{2}\left(\varphi+\theta_{i}\right)
$$

where $E$ is the amplitude of the linearly polarized light and $\varphi$ represents the angle between the CMOS device and the analyzer. The sampling frequency of the magneto-optical sensor with a CMOS camera is 50 frames per second, and the image resolution of CMOS camera is 512 pixel $\times 512$ pixel.

The light intensity $I_{i}(i=1,2,3)$ can be described as follows

$$
\begin{aligned}
& I_{1}=E^{2} \cos ^{2}\left(\varphi+\theta_{1}\right) \\
& I_{2}=E^{2} \cos ^{2}\left(\varphi+\theta_{2}\right) \\
& I_{3}=E^{2} \cos ^{2}\left(\varphi+\theta_{3}\right)
\end{aligned}
$$

the light intensities $I_{1}, I_{2}$ and $I_{3}$ representing areas 1, 2 and 3 seem to be in black, brown and orange, respectively, as is shown in Figure 1.

\subsection{Magnetic Domain Theory}

Q235 is ferromagnetic material and is widely used in automotive, shipbuilding, bridge and pressure vessel industry, which can be easily magnetized by electromagnets [31]. Weiss firstly put forward the magnetic domain theory based on the quantum theory, explicating the magnetization mechanism of ferromagnetic material in the microscopic aspect [32]. In Figure 2, a spontaneous magnetization region within a magnetic material is called the magnetic domain, and the interface between each domain is called the domain wall. The directions of magnetic domains are different, but the effect of the entire magnetic 
domain is offset each other, and the magnetic torque of the whole object is zero; accordingly, the weldment shows no magnetism and can't attract other magnetic materials. However, the magnetization is in a uniform direction in the magnetic domain when the material is magnetized under the external magnetic field. When the magnetic field intensity changes periodically, the closed magnetization curve of strong magnetic materials is called hysteresis loop, which reveals the hysteresis phenomenon of ferromagnetic material magnetization. The relation between magnetic induction $B$ and magnetic field $H$ can be expressed as

$$
B=u H
$$

where $u$ is the magnetic permeability for the material, the permeability depends on the $H$ field.

without the external magnetic field with the effect of extra magnetic field

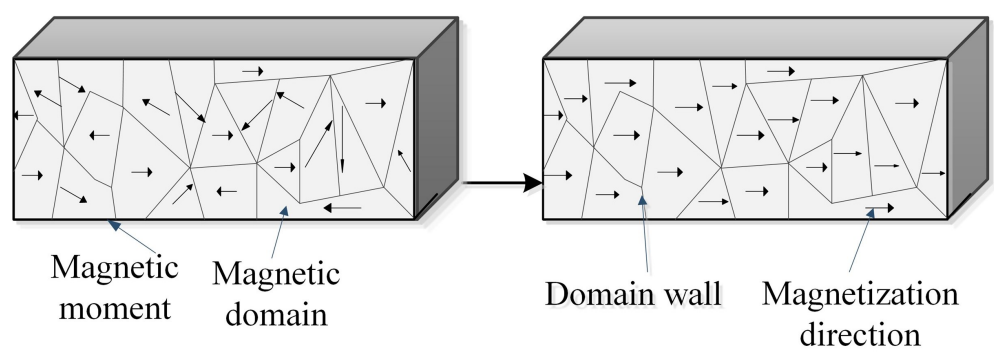

Figure 2. Theory of magnetic domain.

\subsection{Material and Laser Welding Parameters.}

Ferromagnetic specimen Q235 with a size of $150 \times 50 \times 2 \mathrm{~mm}^{3}$ (length $\times$ width $\times$ thickness) was welded by Nd: YAG laser welding machine in the argon atmosphere, and Nd: YAG laser was employed to join the butt joint. The specimen surface was smooth and clean and the chemical composition of Q235 is shown in Table 1. After repeated welding tests, we have determined the appropriate welding parameters that will not produce defects. The welding parameters including peak power, welding speed, defocusing, average power, and pulse duration, are shown in Table 2. After welding, The welded sample was cut into fittingly sized samples of $20 \times 20 \times 2 \mathrm{~mm}^{3}$ (length $\times$ width $\times$ thickness) by wire-electrode cutting. Then the cut samples were ground sequentially with $800^{\#}, 1000^{\#}, 1200^{\#}, 1500^{\#}, 2000^{\#}$, and $2500^{\#}$ grinding paper and were polished to $0.5 \mu \mathrm{m}$ with grinding and polishing machine. Thereafter, the grinded surface was etched in a solution of $1 \mathrm{~mL}$ nitric acid and $24 \mathrm{~mL}$ ethanol to acquire desirable observations. The welded joint microstructure of etching and grinding samples was observed by SEM, and further investigated the differences of welded joint microstructure in various weld regions.

Table 1. Chemical Composition of Q235 (wt.\%).

\begin{tabular}{ccccccc}
\hline $\mathbf{C}$ & $\mathbf{S i}$ & $\mathbf{M n}$ & $\mathbf{P}$ & $\mathbf{S}$ & $\mathbf{N b}$ & $\mathbf{B}$ \\
\hline 14 & 19 & 55 & 2.8 & 2.0 & \multicolumn{2}{c}{ Remainder } \\
\hline
\end{tabular}

Table 2. Laser Welding Parameters of Each Group.

\begin{tabular}{ccccc}
\hline $\begin{array}{c}\text { Peak Power } \\
(\mathbf{k W})\end{array}$ & $\begin{array}{c}\text { Speed } \\
(\mathbf{m} / \mathbf{m i n})\end{array}$ & $\begin{array}{c}\text { Defocusing } \\
(\mathbf{m m})\end{array}$ & $\begin{array}{c}\text { Average Power } \\
\mathbf{( W )}\end{array}$ & $\begin{array}{c}\text { Pulse Duration } \\
(\mathbf{m s})\end{array}$ \\
\hline 1.5 & 5 & 1 & 114.5 & 6 \\
\hline
\end{tabular}




\section{Experimental Set-Up}

As demonstrated in Figure 3, the experimental installation consists of a three-axis moving table, electromagnetic yoke, direct-current power supply, industrial control computer, weldment, magnetic optical sensor, and image acquisition system. The three-axis moving table is moved by an industrial control computer. The intensity of the magnetic field is controlled by putting direct-current signal into the electromagnetic yoke, and the weldment was magnetized under the constant magnetic field. MO images of welded joint were collected by the MO sensor with CMOS camera under constant magnetic field.

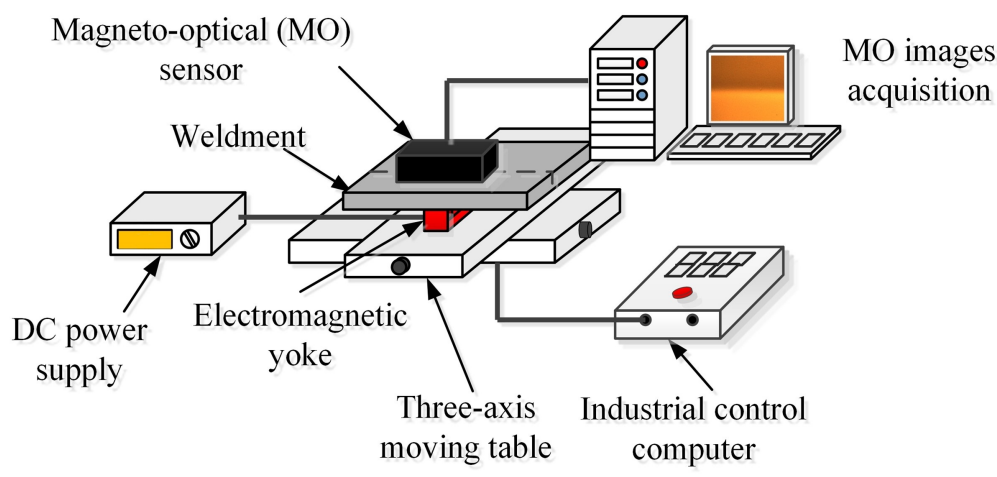

Figure 3. Experimental setup to investigate the welded joint microstructure based on magnetooptical imaging.

\section{Magnetic Dipole Model of Welded Joint}

In order to study the changes of the magnetic field in different microstructures more clearly, we established a finite element model of the welded joint, as shown in Figure 4. The finite element model is divided into three regions, the outermost layer is $B M$, the middle layer is HAZ, and the innermost layer is WZ.

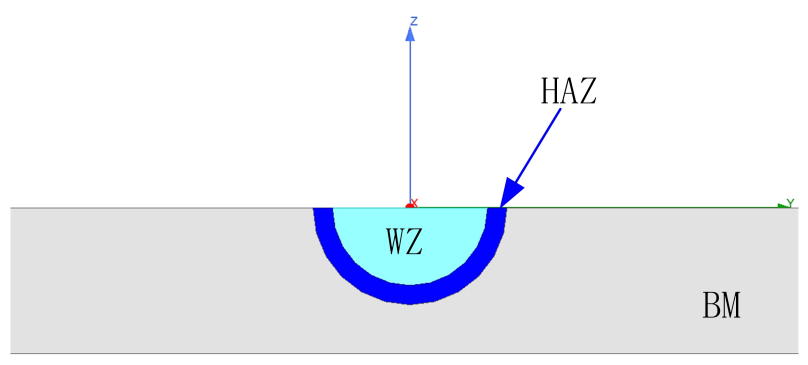

Figure 4. The simulation model of welded joint for thin plate.

The permeability of these three areas has been set accordingly. The permeability of $\mathrm{BM}$ is $400\left(u_{B M}\right)$, the permeability of HAZ is $350\left(u_{H A Z}\right)$, and the permeability of WZ is $300\left(u_{W Z}\right)$. By applying the same external magnetic field, the influence of external magnetic field on the welded joints is observed, and the weak variation in magnetic permeability is studied. Through the analysis results of the finite element analysis software, we can observe this change more directly.

We carried out two sets of simulation experiments here. In the first group, the BM that has not been welded is excited by a DC magnetic field. The magnetic field distribution directly above the steel plate is obtained. The material was then changed to HAZ and a repeat analysis was performed. The results of the two analyses is compared. In the second group, the same simulation as BM was performed on the steel plate containing HAZ and WZ again. The results were compared with the first group of BM. 


\section{Result and Discussion}

\subsection{Characteristics of Mo Images of Welded Joint}

As is shown in Figure 5, the weld seam and corresponding MO images of welded joint surface are included. With regard to extrinsic feature observed in the real image of the welded joint, the welding quality without was not good and the weld didn't form organized scaly, because the average power was high and the laser welding speed was quick. However, the colour and lightness of MO images made a big difference on both sides of the weld, and the lightness on the right is much brighter than that on the left.

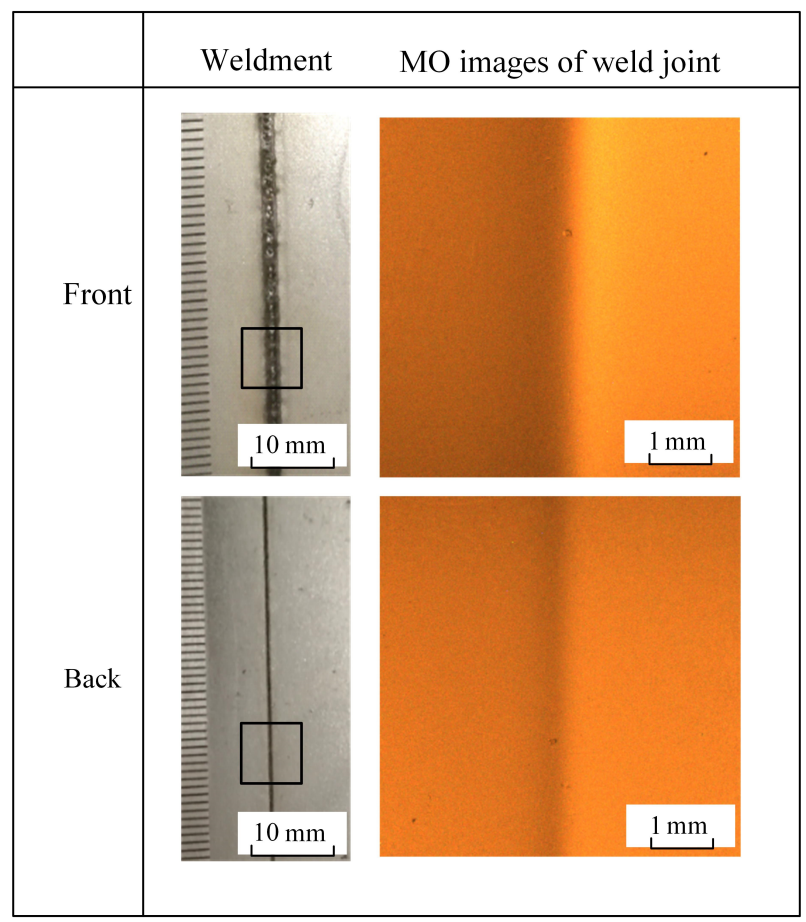

Figure 5. Real figures and magneto-optical diagrams of the welded seam.

Due to the noise interference during the acquisition process, MO images of the weld seam was denoised by average filtering to acquire useful information. Then the pixels of row 256 of MO images were shown in Figure 6, which present the variation of MO image on the topside of the weld seam. In general, the gray curve is approximately for the weld seam. The gray curve is divided into three regions, including A1 (A1L, A1R), A2 (A2L, $\mathrm{A} 2 \mathrm{R})$, and $\mathrm{A} 3$ (A3L, A3R). As the findings show, the gray scales of A1 increase sharply from 0.46 to 0.65 ; the curve width of $\mathrm{A} 2$ is relatively narrow due to the quick heating and solidification processes during laser welding, and the gray scales of A3 appear a stable fluctuation. In region A3, the gray value gradually decreased from 0.47 to 0.42 on the left; it decreased from 0.69 to 0.64 in region A3R. In region A2, the gray value fluctuated within a small range from 0.41 to 0.46 and from 0.65 to 0.7 on the left and right sides of the weld center, respectively. In region A1, the gray curve shows a sharply increasing tendency from left to right. Additionally, the gray value of region A1L increased from 0.46 to 0.55 on the left side of the weld, and increased dramatically from 0.55 to 0.65 on the right. Moreover, the gray value variations on the left are consistent with that on the right of $A 1, A 2$, and A3, respectively. The brightness of A2 is brighter than that of A1 and A3 on the right side, and the brightness of $\mathrm{A} 2 \mathrm{~L}$ is darker than that of A1L and A2L.

The pixels of row 256 of the welded joint on the back was chosen to be shown in Figure 7, and the variation of the gray curve is similar to that on the front side of the weld seam. Similarly, the variation of the gray value can be divided into three regions, a1(a1L, a1R), a2(a2L, a2R), and a3(a3L, a3R). In region a3, the gray value decreased from 0.55 to 0.48 on the left and from 0.61 to 0.58 on the right. In region a2, the gray value fluctuated within 
a small range increasing from 0.47 to 0.49 and from 0.57 to 0.617 on the left and right sides, respectively. In region a1, the gray value increased sharply from left to right. In addition, the gray value of region a1L increased from 0.49 to 0.53 on the left, and increased abruptly from 0.53 to 0.57 in region a1R. Similarly, the brightness of a1L is brighter than that of a2L on the left side of the weld, but the brightness of a1R is darker than that of a2R on the right. As a whole, in the region of a1, a2 and a3 of weld, the gray value variations on the left side remained similar to those on the right side. These three areas (A1-3 and a1-3) are likely to be related to the three regions of the welded joint.

the pixel of row 256
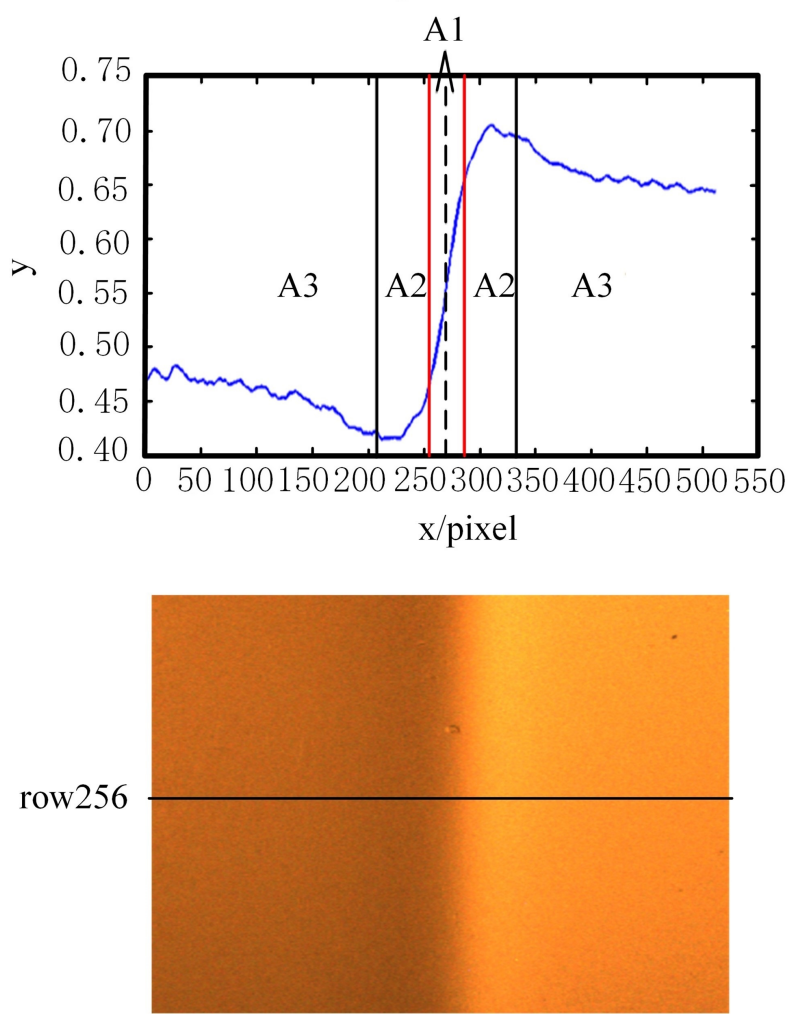

Figure 6. The pixels of row 256 of the welded joint on the topside and the corresponding MO image.

\subsection{Comparison of MO Microstructure and SEM Images of Welded Joint}

As is shown in Figure 8, high temperature resulted in some deformation of the microstructure of material during the laser welding process, and there are great differences in $\mathrm{MO}$ images of welded joint microstructure. In Figure 8, comparing graphics of diverse regions $(1,2$, and 3$)$ of weldment are illustrated, including the MO images of the welded joint surface and section, and the microstructure images of the section. The areas of welded joint section include the weld zone (WZ), the heat-affected zone (WAZ), and the base material (BM), then separated by red and black lines, respectively. As is shown in Figure 8a-d, region 1 is dark brown and surrounded by a blue arc, respecting the WZ. Region 3 is the orange area covering the outside of the black arc, and there is BM. Region 2 is HAZ, existing on both sides of region 1 , and its colour is light brown; the areas of region 2 are the narrowest of all the regions, and the colour of region 2 is between those of the WZ and BM. The three zones possess respectively the characteristics of WZ, HAZ, and BM, as depicted in Figure $8 \mathrm{f}-\mathrm{h}$. As the microstructure images show, the grains are lath-shaped and coarse in the $\mathrm{WZ}$, and the distribution of grains is dense and inhomogeneous. There is a clear boundary near the WZ and the HAZ, and the isometric and columnar grains exist at the edge of the HAZ and they are distributed inhomogeneously. The grains of the $\mathrm{BM}$ are the finest among the three regions, containing ferrite and pearlite, and distributed 
homogeneously. The grains of the BM are the finest of the three regions, containing ferrite and pearlite, and distributed homogeneously.
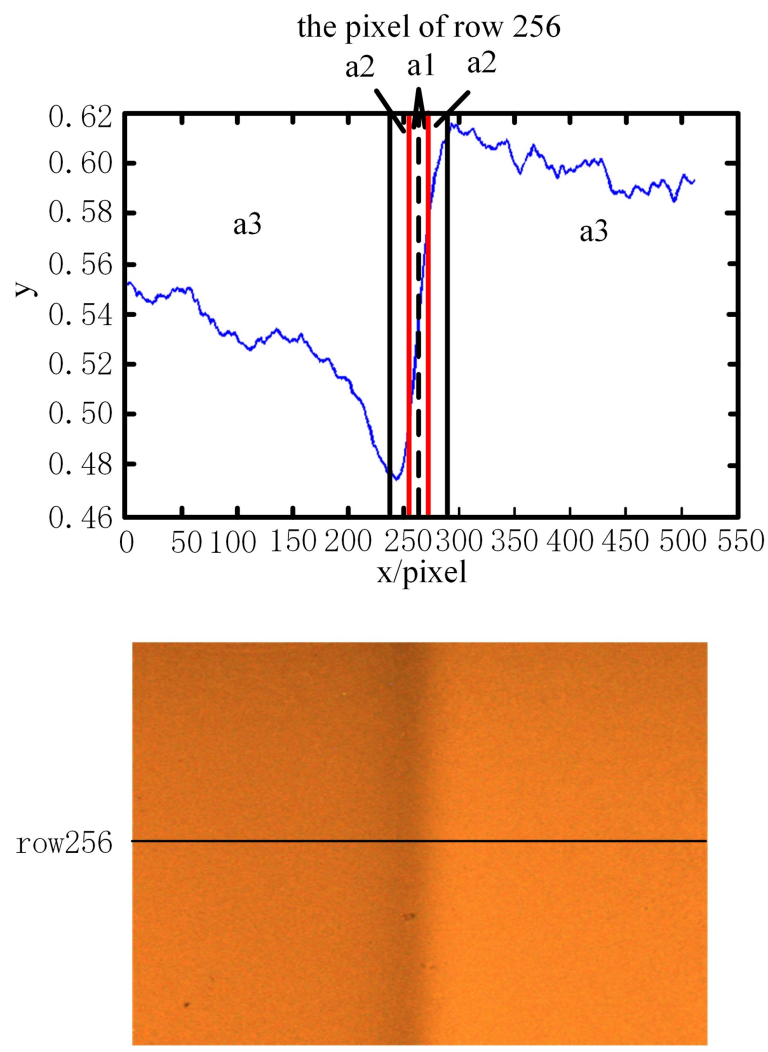

Figure 7. The pixels of row 256 of the welded joint on the topside and the corresponding MO image.

Figure 9 illustrates the magnification of welded joint microstructures observed by SEM. Although these images are not as clear as the grain boundaries of the martensite and pearlite grains found in other metallurgical products, it is still possible to distinguish three regions including the weld zone, the heat-affected zone, and the base material of the welded joint. Figure $9 \mathrm{c}-\mathrm{e}$ represent the welded joint microstructure of the WZ, HAZ and $\mathrm{BM}$, respectively. And Figure $9 \mathrm{a}, \mathrm{b}, \mathrm{g}$ are the magnifications of corresponding regions. As is shown in Figure 9a,c, the weld zone exhibits columnar grains, which are coarser than that of the BM. During the welding process, the temperature of the WZ was overheated, whose gains growth rapidly caused thick martensite. The temperature of the HAZ was lower than that of the WZ, afterward, the weld was cooled quickly, and the austenite transforms into martensite. Hence the grains of the WZ were finer than those of the HAZ. Figure 9d,b exhibits the columnar grains in HAZ, among which the isometric grains were detected at the boundary of the WZ and HAZ. As illustrated in Figure 9e,g, base mental is far from the weld zone and BM is on the sides of the weld are almost unaffected by the welding heat treatment, then the grains of the BM are unchanged almost. Hence, the grains are smaller and finer than those of the WZ and HAZ. And Figure 9f shows the boundary of the welding zone and the heat-affected zone, in which the grain size becomes increasingly coarser upon moving from the HAZ to the WZ side. During the laser welding process, the unmelted base metal served as the substrate for nucleation, and afterward, the grains' growth proceeded toward the base mental side, then the columnar grains were generated [33].

The magnetism of welded was influenced by the changes of microstructure during the laser welding process. With the decreasing of grain size, the permeability of the materials decreases and the coercivity increases gradually; then the materials are difficult to be demagnetized, and greater residual magnetism remains. In general, the magnetic induction intensity of the welds decreases with the decrease of grain size. Figure 10 shows the relationship between magnetic induction intensity $B$ and magnetic intensity 
$H$ of ferromagnetic materials with different microstructure sizes. It can be seen from the curve that the smaller the grain size, the weaker the magnetic induced intensity. Due to the variant microstructure in different regions, the magnetic induction intensity $B$ of welded is different when the external magnetic field is provided. Parameter $S$ is defined as the grain size; $S_{M}, S_{H}$, and $S_{B}$ are the grain size in the $W Z, H A Z$ and $B M$, respectively. The microstructure size can be observed as $S_{B}>S_{H}>S_{M}$ from the MO images of different regions, so it can be concluded that $B_{W Z}<B_{H A Z}<B_{B M}$, where $B_{W Z}, B_{H A Z}, B_{B M}$ are the magnetic induction intensity of $W Z, H A Z$ and $B M$, respectively.

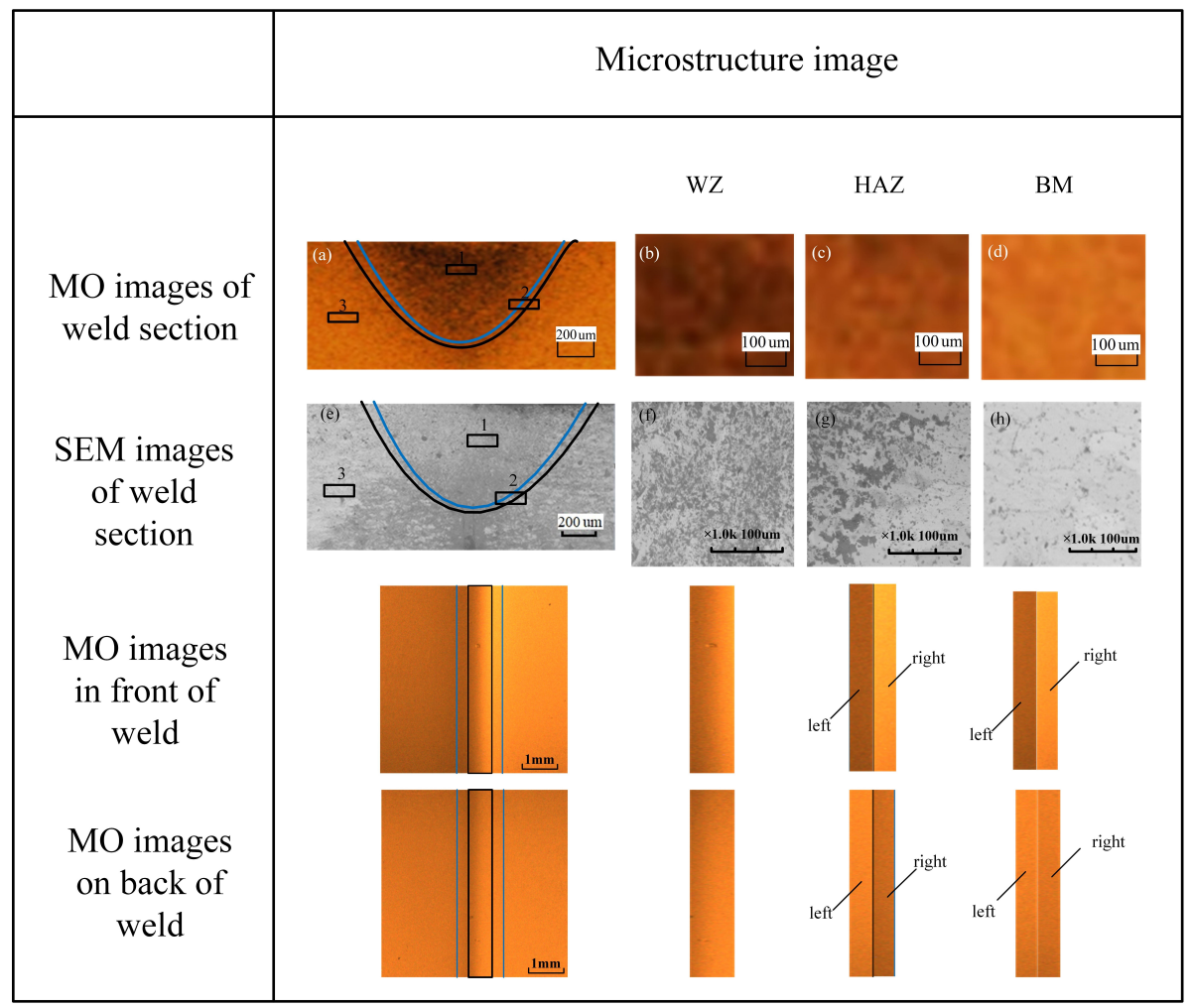

Figure 8. The comparisons of MO and SEM images of welded seam microstructure in different regions (including the magneto-optical image of the weld seam surface and cross-section, partial selected image of weld seam surface in magneto-optical image, the SEM image).

Due to the excitation of the applied external magnetic field's $S$ pole, the color on the left is darker than that on the right in the surface MO image. From Formula (2),

$$
\begin{aligned}
& I_{1(A 1 L)}=E^{2} \cos ^{2}\left(\varphi+\theta_{A 1 L}\right) \\
& I_{1(A 2 L)}=E^{2} \cos ^{2}\left(\varphi+\theta_{A 2 L}\right)
\end{aligned}
$$

Then, from Formula (1),

$$
\begin{aligned}
& \theta_{(A 1 L)}=V\left(B_{A 1 L}\right) \\
& \theta_{(A 2 L)}=V\left(B_{A 2 L}\right)
\end{aligned}
$$

where $\theta_{A 1 L}, B_{A 1 L}$ and $I_{1(A 1 L)}$ are polarization angle, magnetic induction intensity, and light intensity in region $\mathrm{A} 1$ on the left side of the seam, respectively. And $\theta_{A 2 L}, B_{A 2 L}$ and $I_{1(A 2 L)}$ are correspondingly in region A2 on the left side of the seam. From Formulaes (4) and (5), the brighter the MO images, the smaller the Faraday angle; then from Formulaes (6) and (7), the smaller the Faraday angle, the weaker the magnetic induction intensity. And the weaker the magnetic induction intensity is, the smaller the grain size. 

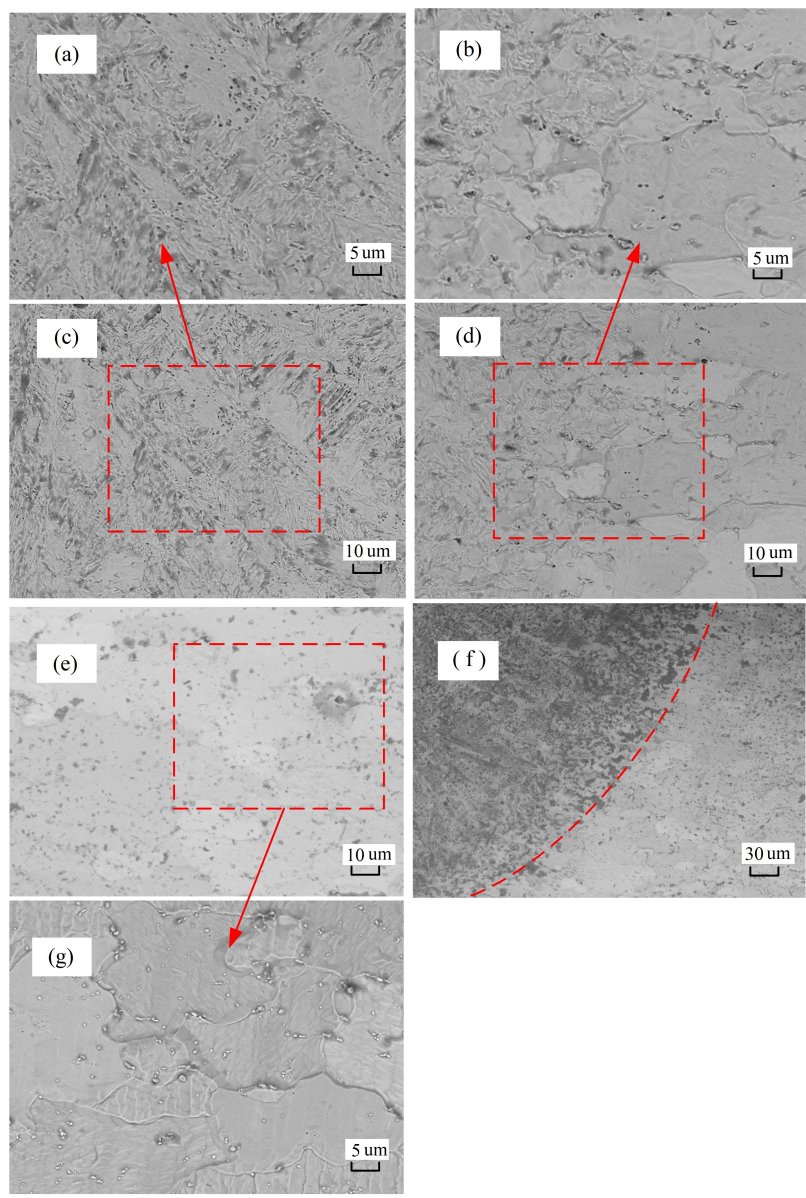

Figure 9. SEM images of welded joint section in different regions: $(\mathbf{a}, \mathbf{c})$ the welding zone with different magnifications; (b,d) the heat affected zone with different magnifications; $(\mathbf{e}, \mathbf{g})$ the base metal with different magnifications; (f) the boundary of welding zone and heat affected zone.

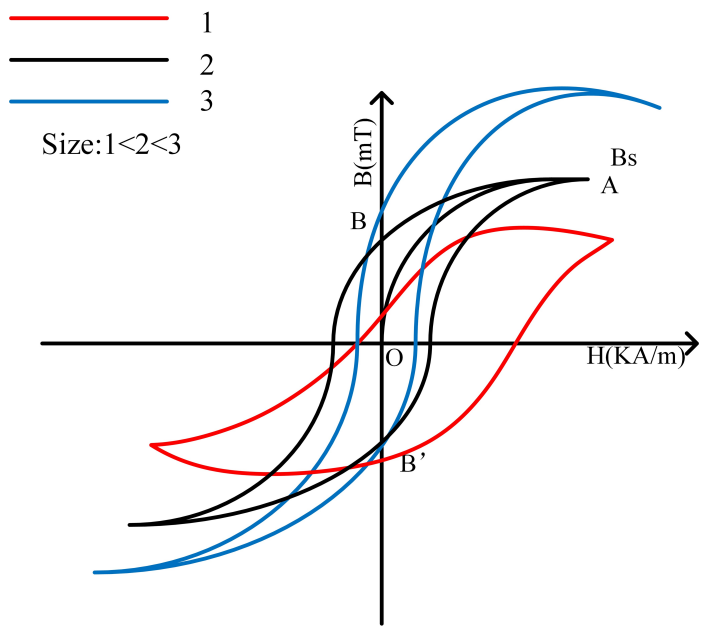

Figure 10. Magnetizing curve of ferromagnetic materials in different microstructure size.

Similarly, it can be seen that magnetic induction intensity $B_{A 1 L}$ is weaker than $B_{A 2 L}$ on the left side of seam line, while the light intensity $I_{1(A 1 L)}$ is brighter than $I_{1(A 2 L)}$, and the polarization angle $\theta_{A 1 L}$ is smaller than $\theta_{A 2 L}$ on the left of weld. Furthermore, the light intensity $I_{2(A 1 R)}$ is darker than $I_{2(A 2 R)}$, the polarization angle $\theta_{A 1 R}$ is smaller than $\theta_{A 2 R}$, and $B_{A 1 R}$ is weaker than $B_{A 2 R}$ on the right of the seam line. Hence, the brightness $I_{2(A 1 R)}$ of the region $\mathrm{A} 1$ on the right of the weld seam is darker than that of $I_{2(A 2 R)}$, where $\theta_{A 1 R}, B_{A 1 R}$ and $I_{2(A 1 R)}$ are polarization angle, magnetic induction intensity, and light 
intensity in region $\mathrm{A} 1$ on the right of the seam line, respectively, and $\theta_{A 2 R}, B_{A 2 R}$, and $I_{2(A 2 R)}$ are correspondingly in region A2 on the right of the seam line. Based on the results, the difference of microstructure of welded joint can be evaluated by $\mathrm{MO}$ imaging method.

\subsection{Magnetic Field Intensity Distribution of Welded Joint Simulation Model}

The first set of simulation results is shown in the Figure 11. Figure 11a is the overall magnetic field distribution curve of the steel plate surface. The green box is the welded joint and the enlarged view is shown in Figure 11b. It can be seen from the figure, when the material parameter changes from BM to HAZ, since $u_{H A Z}<u_{B M}$, his is because of the high permeability of the steel plate, more magnetic field enters the steel plate, and the external distribution of the excitation field is reduced. At the same time, since there is no welded joint part, there is no magnetic field change, and the external magnetic field in this area presents a linear distribution.
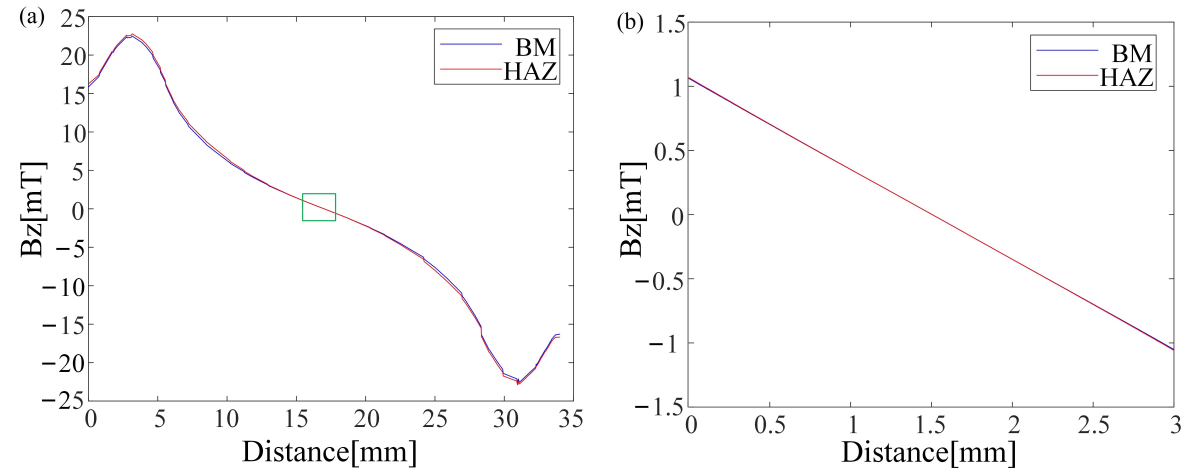

Figure 11. Magnetic field distribution under different permeability of BM and HAZ. (a) Overall distribution map. (b) Distribution map of the green box.

The second set of simulation experiments is shown in Figure 12. Due to the addition of the welded joint part, the magnetic field distribution in the welded joint area has been distorted. From Figure 12b, it can be seen that there are two sharply changing regions. This is because the three regions of the finite element model are not smoothly transitioned. The boundary of the magnetic domain appears when the magnetic permeability abruptly changes, and polarization appears at the boundary. Microscopically, it can be understood as the appearance of the accumulation of magnetic domains and the formation of new small magnets. However, the change of magnetic permeability makes part of the magnetic field unable to directly penetrate into the new region, resulting in magnetic field distortion. This distorted magnetic field is opposite to the external magnetic field, thus weakening the external magnetic field and making the strength curve of HAZ weaker than that of BM. This means that the leakage magnetic field in this area is stronger. similarly, the boundary between WZ and HAZ shows the same phenomenon, which further weakens the strength of the external magnetic field.

Through simulation, it is further verified that when the permeability is reduced, the leakage magnetic field will increase, which is consistent with our experimental results. 

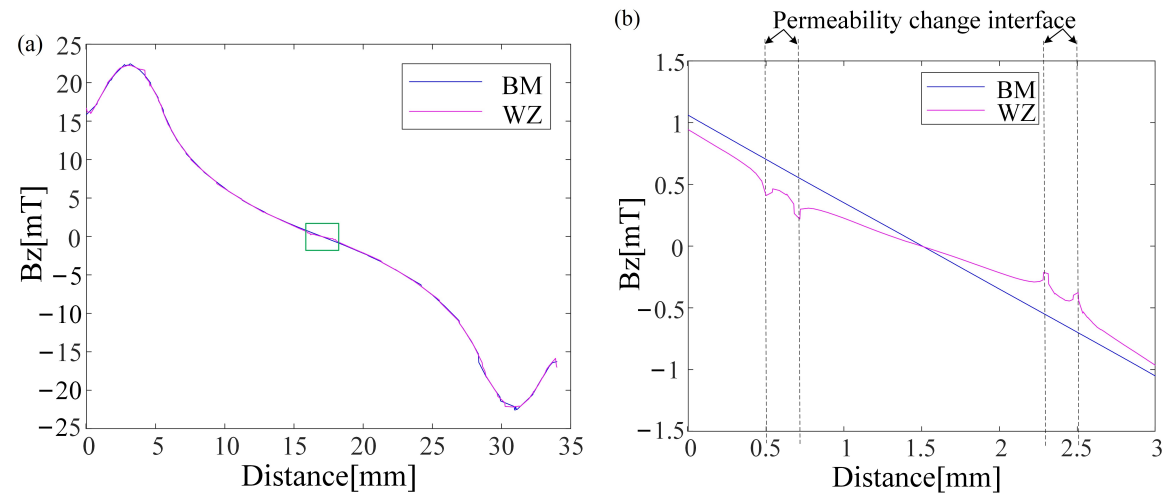

Figure 12. Simulation comparison chart of BM and welded joint part. (a) Overall distribution map. (b) Distribution map of the green box.

\section{Conclusions}

This paper has proposed a novel approach for researching microstructure of welded joints using MO imaging method under a constant magnetic field. And the microstructure of welded joint was studied based on Faraday effect. The MO images of the weld were processed by image processing technology to extract the grayscale curves of the rows of interest for analysis. Furthermore, the SEM images of welded joint microstructure have been analyzed and verified the feasibility of the results of the MO images. The results show that the MO imaging method is effective for researching the microstructure of the welded joint, and the key conclusions derived from this investigation are as follows:

According to the characteristic of light intensities, colours, and grain sizes, three regions containing the $\mathrm{WZ}, \mathrm{HAZ}$, and $\mathrm{BZ}$ were identified in the section of the welded joint.

In $\mathrm{WZ}$, the light intensity of the $\mathrm{MO}$ image is the darkest, and the grains are columnar mainly. The mixture of isometric and columnar grains are recognized in the HAZ, which resulted from the fast solidification and different deformation of the microstructure. In the $\mathrm{BM}$ zone, the light intensity is the brightest, and the grains are the finest.

With the decrease of grain size, the permeability of the material decreases and the strength of light $\mathrm{MO}$ weakens. The relationship between grain sizes of ferromagnetic materials and magnetic induction intensity is of great significance to the change of welded joint microstructure. As a result, $\mathrm{MO}$ imaging is an effective approach for microstructure investigation of welded joint microstructure under a constant magnetic field.

Author Contributions: Conceptualization, X.G.; data curation, C.W. (Chuncao Wang), X.G., M.T. and Y.Z.; formal analysis, C.W. (Congyi Wang), C.W. (Chuncao Wang) and M.T.; funding acquisition, X.G. investigation, C.W. (Congyi Wang) and C.W. (Chuncao Wang); methodology, C.W. (Congyi Wang), X.G. and Y.Z.; project administration, X.G.; resources, X.G.; supervision, C.W. (Congyi Wang), X.G. and Y.Z.; validation, C.W. (Congyi Wang), M.T., X.G. and Y.Z.; visualization, C.W. (Congyi Wang) and C.W. (Chuncao Wang) ; writing - original draft preparation, C.W. (Congyi Wang), C.W. (Chuncao Wang), X.G. and Y.Z.; writing-review and editing, X.G. All authors have read and agreed to the published version of the manuscript.

Funding: This work was partly supported by the Guangzhou Municipal Special Fund Project for Scientific and Technological Innovation and Development (Grant No. 202002020068, 202002030147).

Institutional Review Board Statement: Not applicable.

Informed Consent Statement: Not applicable.

Data Availability Statement: Not applicable.

Conflicts of Interest: The authors declare no conflict of interest. 


\section{References}

1. Zhang, Y.; You, D.; Gao, X.; Katayama, S. Online monitoring of welding status based on a DBN model during laser welding. Engineering 2019, 5, 671-678. [CrossRef]

2. Gao, X.; Sun, Y.; Katayama, S. Neural network of plume and spatter for monitoring high-power disk laser welding. Int. J. Precis. Eng. Manuf.-Green Technol. 2014, 1, 293-298. [CrossRef]

3. Riofrío, P.G.; Ferreira, J.A.; Capela, C.A. Imperfections and modelling of the weld bead profile of laser butt joints in HSLA steel thin plate. Metals 2021, 11, 151. [CrossRef]

4. Oliveira, J.P.; Shen, J.; Zeng, Z.; Park, J.M.; Choi, Y.T.; Schell, N.; Maawad, E.; Zhou, N.; Kim, H.S. Dissimilar laser welding of a CoCrFeMnNi high entropy alloy to 316 stainless steel. Scr. Mater. 2022, 206, 114219. [CrossRef]

5. Oliveira, J.; Schell, N.; Zhou, N.; Wood, L.; Benafan, O. Laser welding of precipitation strengthened Ni-rich NiTiHf high temperature shape memory alloys: Microstructure and mechanical properties. Mater. Des. 2019, 162, 229-234. [CrossRef]

6. Oliveira, J.P.; Shen, J.; Escobar, J.; Salvador, C.; Schell, N.; Zhou, N.; Benafan, O. Laser welding of H-phase strengthened Ni-rich NiTi-20Zr high temperature shape memory alloy. Mater. Des. 2021, 202, 109533. [CrossRef]

7. Zou, P.; Zhang, H.; Lei, M.; Cheng, D.; Huang, S.; Yang, F. Interfacial Microstructure and Formation of Direct Laser Welded CFRP/Ti-6Al-4V Joint. Metals 2021, 11, 1398. [CrossRef]

8. Jin, Y.; Li, Y.L.; Zhang, H. Microstructure and mechanical properties of pulsed laser welded Al/steel dissimilar joint. Trans. Nonferrous Met. Soc. China 2016, 26, 994-1002.

9. Maziarz, W.; Czaja, P.; Chulist, R.; Wójcik, A.; Żrodowski, Ł.; Morończyk, B.; Wróblewski, R.; Kowalczyk, M. Microstructure and Magnetic Properties of Selected Laser Melted Ni-Mn-Ga and Ni-Mn-Ga-Fe Powders Derived from as Melt-Spun Ribbons Precursors. Metals 2021, 11, 903. [CrossRef]

10. Nykyforchyn, H.; Student, O.; Markov, A. Abnormal manifestation of the high-temperature degradation of the weld metal of a low-alloy steel welded joint. Mater. Sci. 2007, 43, 77-84. [CrossRef]

11. Xiong, J.; Yang, X.; Lin, W.; Liu, K. Effects of welding parameters on microstructure and mechanical properties of underwater wet friction taper plug welded pipeline steel. Weld. World 2019, 63, 11-22. [CrossRef]

12. Wu, S.; Han, B.; Zhao, D.; Ji, Y.; Shao, L. Effect of post weld heat treatment on microstructure and fracture toughness of friction welded joint. J. Wuhan Univ. Technol.-Mater. Sci. Ed. 2016, 31, 1347-1351. [CrossRef]

13. Huaming, W.; Dong, L. Microstructure and mechanical properties of AF1410 ultra-high strength steel using laser additive manufacture technique. Chin. J. Lasers 2016, 43, 59-65.

14. Kozhevnikov, V.Y.; Kharazov, V. Metallographic analysis of the welded joint in automatic argon-shielded non-consumable electrode welding. Weld. Int. 2017, 31, 951-953. [CrossRef]

15. Zhang, H.; Li, J.; Guan, Z.; Liu, Y.; Qi, D.; Wang, Q. Electron beam welding of Nimonic 80A: Integrity and microstructure evaluation. Vacuum 2018, 151, 266-274. [CrossRef]

16. Alam, F.; Ahmad, M.; Zafar, M.; Shinwari, M.I.; Luqman, M.; Novruz, N.E.; Sultana, S.; Ullah, F.; Ahmad, M. Intraspecific variation in spermoderm pattern of tribe Acacieae (Mimosoideae) using scanning electron microscopy techniques. Microsc. Res. Tech. 2019, 82, 114-121. [CrossRef] [PubMed]

17. Dangwei, Z.; Jingwen, Z.; Xun, H. X-ray diffraction from self-originized quantum-dots. Acta Photonica Sin. 2002, $31,837$.

18. Chapman, M.; Callahan, P.; De Graef, M. Determination of sample surface topography using electron back-scatter diffraction patterns. Scr. Mater. 2016, 120, 23-26. [CrossRef]

19. Cao, X.; Zhu, P.; Ding, X.; Lu, Y.; Shoji, T. An investigation on microstructure and mechanical property of thermally aged stainless steel weld overlay cladding. J. Nucl. Mater. 2017, 486, 172-182. [CrossRef]

20. Bulgarevich, D.S.; Tsukamoto, S.; Kasuya, T.; Demura, M.; Watanabe, M. Pattern recognition with machine learning on optical microscopy images of typical metallurgical microstructures. Sci. Rep. 2018, 8, 2078. [CrossRef]

21. Winiczenko, R.; Kaczorowski, M. Friction welding of ductile cast iron using interlayers. Mater. Des. 2012, 34, 444-451. [CrossRef]

22. Winiczenko, R.; Kaczorowski, M.; Skibicki, A. The microstructures, mechanical properties, and temperature distributions in nodular cast iron friction-welded joint. J. Braz. Soc. Mech. Sci. Eng. 2018, 40, 347. [CrossRef]

23. Wanrong, G.; Chunkan, T.; Xiaochun, Y. Relationship between the two dimensional resolution and signal-to-noise ratio of laser confocal microscopy. Acta Photonica Sin. 2002, 31, 730.

24. Ma, G.; Wu, D.; Niu, F.; Zou, H. Microstructure evolution and mechanical property of pulsed laser welded Ni-based superalloy. Opt. Lasers Eng. 2015, 72, 39-46. [CrossRef]

25. Gao, X.; Mo, L.; Xiao, Z.; Chen, X.; Katayama, S. Seam tracking based on Kalman filtering of micro-gap weld using magneto-optical image. Int. J. Adv. Manuf. Technol. 2016, 83, 21-32. [CrossRef]

26. Gao, X.; Du, L.; Ma, N.; Zhou, X.; Wang, C.; Gao, P.P. Magneto-optical imaging characteristics of weld defects under alternating and rotating magnetic field excitation. Opt. Laser Technol. 2019, 112, 188-197. [CrossRef]

27. Gao, X.; Ma, N.; Du, L. Magneto-optical imaging characteristics of weld defects under alternating magnetic field excitation. Opt. Express 2018, 26, 9972-9983. [CrossRef]

28. Gao, X.; Ding, D.; Bai, T.; Katayama, S. Weld-pool image centroid algorithm for seam-tracking vision model in arc-welding process. IET Image Process. 2011, 5, 410-419. [CrossRef]

29. Xie, Z.; Gao, X. Edge detection of weld image based on Canny operator. Trans.-China Weld. Inst. 2006, $27,29$. 
30. Gao, X.; Zhen, R.; Xiao, Z.; Katayama, S. Modeling for detecting micro-gap weld based on magneto-optical imaging. J. Manuf. Syst. 2015, 37, 193-200. [CrossRef]

31. Wang, J.; Ma, N.; Murakawa, H.; Teng, B.; Yuan, S. Prediction and measurement of welding distortion of a spherical structure assembled from multi thin plates. Mater. Des. 2011, 32, 4728-4737. [CrossRef]

32. Ducharne, B.; Le, M.Q.; Sebald, G.; Cottinet, P.; Guyomar, D.; Hebrard, Y. Characterization and modeling of magnetic domain wall dynamics using reconstituted hysteresis loops from Barkhausen noise. J. Magn. Magn. Mater. 2017, 432, 231-238. [CrossRef]

33. Ke, W.; Bu, X.; Oliveira, J.; Xu, W.; Wang, Z.; Zeng, Z. Modeling and numerical study of keyhole-induced porosity formation in laser beam oscillating welding of 5A06 aluminum alloy. Opt. Laser Technol. 2021, 133, 106540. [CrossRef] 